\title{
Close relationship between coral-associated Chromera strains despite major differences within the Symbiodiniaceae
}

Amin R. Mohamed ${ }^{1,2,3,4,5^{*}}$, Cheong Xin Chan ${ }^{6,7}$, Mark A. Ragan ${ }^{6}$, Jia Zhang ${ }^{3}$, Ira

Cooke $^{3}$, Eldon E. Ball ${ }^{2,8}$, David J. Miller ${ }^{2,3^{*}}$

\section{Affiliations:}

${ }^{1}$ CSIRO Agriculture and Food, Queensland Bioscience Precinct, St Lucia Brisbane QLD 4067, Australia

${ }^{2}$ ARC Centre of Excellence for Coral Reef Studies, James Cook University, Townsville 4811, Queensland, Australia

${ }^{3}$ Molecular and Cell Biology, James Cook University, Townsville 4811, Queensland, Australia

AIIMS@JCU, Australian Institute of Marine Science, Department of Molecular and Cell

Biology, James Cook University, Townsville 4811, Queensland, Australia

${ }^{5}$ Zoology Department, Faculty of Science, Benha University, Benha 13518, Egypt

${ }^{6}$ Institute for Molecular Bioscience, The University of Queensland, Brisbane, QLD 4072 ,

Australia

${ }^{7}$ School of Chemistry and Molecular Biosciences, The University of Queensland, Brisbane, QLD 4072, Australia

${ }^{8}$ Division of Ecology and Evolution, Research School of Biology, Australian National University, Acton, ACT 2601, Australia

*correspondence: Dr David Miller Email: david.miller@jcu.edu.au Tel: +61747814473 Fax: +61747816078

Keywords: Chromera, RNA-Seq, transcriptome, symbiosis, Symbiodiniaceae

Running title: Chromera comparative transcriptomics 


\section{Summary}

Reef-building corals live in a mutualistic relationship with photosynthetic algae (family Symbiodiniaceae) that usually provide most of the energy required by the coral host. This relationship is sensitive to temperature stress; as little as a $1^{\circ} \mathrm{C}$ increase often leading to collapse of the association. This sensitivity has led to interest in the potential of more stress tolerant algae to supplement or substitute for the normal Symbiodiniaceae mutualists. In this respect, the apicomplexan-like microalga Chromera is of particular interest due to its greater temperature tolerance. We generated a de novo transcriptome for a Chromera strain isolated from a GBR coral ("GBR Chromera") and compared to those of the reference strain of Chromera ("Sydney Chromera"), and to those of Symbiodiniaceae (Fugacium, Cladocopium and Breviolum), as well as the apicomplexan parasite, Plasmodium falciparum. By contrast with the Symbiodiniaceae, the two Chromera strains had a high level of sequence similarity evident by very low levels of divergence in orthologous genes. Although KEGG categories provide few criteria by which true coral mutualists might be identified, they do supply a molecular rationalization for the ubiquitous association of Cladocopium strains with Indo-Pacific reef corals. The presence of HSP20 genes may underlie the higher thermal tolerance of Chromera.

\section{Introduction}

The ecological success of reef-building corals is generally attributed to their ability to establish mutualistic relationships with specific photosynthetic algae - members of the dinoflagellate family Symbiodiniaceae (formerly, the genus "Symbiodinium"). However, as is now widely appreciated, this relationship breaks down under 
environmental stress, and coral reefs globally are under threat as a consequence of the increasing frequency of weather events that exceed the thermal thresholds of corals (Hughes et al., 2017). In addition to Symbiodiniaceae, a wide range of uncharacterized eukaryotes are associated with corals, including apicomplexanrelated lineages (ARLs) (Clerissi et al., 2018) that can sometimes occur in high abundance (Kwong et al., 2019). Two of these ARLs isolated in association with corals, Chromera velia (Moore et al., 2008) and Vitrella brassicaformis (Obornik et al., 2012), constitute the newly defined phylum Chromerida. As the closest free-living relatives of the parasitic Apicomplexa, these photoautotrophic alveolates are of considerable scientific interest (Moore et al., 2008). Whole genome sequencing (Woo et al., 2015) has recently reaffirmed the close relatedness of Chromera and Vitrella.

The nature of the relationship between corals and Chromera has been a subject of debate. Given its photosynthetic ability (Moore et al., 2008) and its ability to colonize coral larvae (Cumbo et al., 2013), it was initially thought that Chromera might be an alternative coral mutualist, potentially bringing the benefit of higher thermal tolerance than most Symbiodiniaceae (Visser et al., 2012; Chakravarti et al., 2019). However, several lines of evidence now imply otherwise. It has recently been shown that the transcriptomic response of the coral host post Chromera uptake (Mohamed et al., 2018) differed markedly from that of the same coral to a mutualistic strain of Symbiodiniaceae (Mohamed et al., 2016), and resembled the response to incompatible ("incompetent”) Symbiodiniaceae strains (Voolstra et al., 2009). The apparently hostile responses of coral larvae to Chromera during infection suggested that Chromera is more likely to be a parasite or a commensal of corals rather than a 
mutualist (Mohamed et al., 2018). Other lines of evidence support this suggestion (Barott et al., 2011; Janouškovec et al., 2012, 2013), including a recent metaanalysis which implies that Chromera is near exclusively associated with coral biogenous sediments (Mathur et al., 2018).

The present work sought to address two specific issues, in both cases making use of a de novo transcriptome assembly generated for a strain of Chromera isolated from corals on the Great Barrier Reef (GBR). Whilst Chromera was originally isolated from a Sydney Harbor coral, it is known to have a wide distribution (Janouškovec et al., 2012; Visser et al., 2012), and the diversity within this monospecific genus has not been systematically explored. Given the metabolic diversity that is now known to exist (LaJeunesse et al., 2018) within what was previously known as "Symbiodinium", the extent to which conclusions about the coral-Chromera interaction based on the GBR isolate are generalizable is unknown.

The first goal of the present study was therefore to estimate the degree of divergence between the GBR strain of Chromera and that originally isolated from Sydney harbor. Given the evidence that Chromera is unlikely to be a coral mutualist, the second goal was to investigate the repertoires of genes that are thought to play roles in symbiosis and environmental stress tolerance in Chromera and compare these with those of three members of the Symbiodiniaceae. Cladocopium goreaui (formerly Clade $\mathrm{C} 1$ Symbiodinium) was isolated from a colony of Acropora tenuis on the GBR (Howells et al., 2012), and is mutualistic with many Indo-Pacific corals, particularly Acropora species. Breviolum minutum (formerly Clade B Symbiodinium) was isolated from the 
Caribbean coral Orbicella faveolata, and is a mutualist of Caribbean corals.

Fugacium kawagutii (formerly Clade F Symbiodinium) was originally isolated in association with the Hawaiian reef-building coral Montipora verrucosa and the initial whole-genome analyses followed the assumption that Fugacium is a coral mutualist (Lin et al. 2015). However, Fugacium failed to infect juvenile corals (Yuyama et al., 2016), and the consensus now is that Fugacium is probably a surface associate of corals rather than an endosymbiont (Liu el al., 2018; LaJeunesse et al., 2018;

González-Pech et al., 2019). Thus, the expectation was that, with respect to metabolic repertoire, the Chromera strains would resemble Fugacium rather than the known coral mutualists, Cladocopium and Breviolum. Whilst the results suggest that small HSPs may account for the tolerance of Chromera to elevated temperatures, they were inconclusive with respect to the nature of relationships between corals and Chromera or Fugacium. The comparative analyses do, however, provide a molecular rationalization for the near ubiquitous association of Cladocopium with Indo-Pacific corals in general and with Acropora spp in particular.

\section{Experimental procedures}

\section{Chromera culture and culturing conditions}

A culture of Chromera (Mdig3 strain) from the University of Technology Sydney (Cumbo et al., 2013) was used in this study and referred to as "GBR Chromera". The identity of the culture was confirmed both by microscopy and by using Chromeraspecific PCR primers (Supplementary information Figure 1, Table 1). This Chromera strain was originally isolated from the stony coral Montipora digitata (Acroporidae) 
from Nelly Bay, Magnetic Island on the inner central part of the Great Barrier Reef. Cultures were maintained at $25^{\circ} \mathrm{C}$ in Guillard's f/2 medium on a $12 \mathrm{~h} / 12 \mathrm{~h}$ day and night regime. Note that Chromera was subjected to a variety of treatments prior to RNA extraction in order to ensure that the transcriptome assembly captured as many genes as possible. Culture conditions included control, dark stress, cold shock, heat shock, motile and mixotrophic (for details see Supplementary Methods). In all cases, exponentially growing cultures were separated and subjected to the treatment condition and harvested at the end of the experimental treatment. During culturing no antibiotics were used to exclude any potential contribution of the antibiotic treatment to the mRNA expression in the cultures.

\section{RNA isolation and high-throughput sequencing}

$50 \mathrm{~mL}$ of Chromera cultures were pelleted by spinning the cultures at 3,000 $\mathrm{g}$ for 5 min. Pellets were suspended in $1 \mathrm{ml} 0.2 \mu \mathrm{m}$ sterile FSW and centrifuged at 3,000 $\mathrm{g} g$ for 5 min. Pellets were snap frozen in liquid nitrogen and stored at $-80^{\circ} \mathrm{C}$ until further treatment. Total RNA was isolated from $\sim 80 \mathrm{mg}$ of the frozen Chromera pellets using the RNAqueous ${ }^{\circledR}$ Total RNA Isolation Kit (Ambion). The pellets were lysed twice for 20 s at $4.0 \mathrm{~ms}^{-1}$ in Lysing Matrix D tubes (MP Biomedicals, Australia) containing 960 $\mu \mathrm{L}$ of lysis/binding solution plus $80 \mu \mathrm{L}$ of the Plant RNA Isolation Aid (Ambion, USA) on a FastPrep®-24 Instrument (MP Biomedicals, Australia). RNA was bound to filter cartridges supplied with the kit and washed three times, finally RNA was eluted in 40 $\mu \mathrm{L}$ of the elution solution. RNA quantity and quality were assessed using a NanoDrop ND-1000 spectrometer, Qubit® 2.0 fluorometer and Agilent 2100 bioanalyzer. Messenger RNA (mRNA) was isolated from $1 \mu \mathrm{g}$ of total RNA and 6 RNA-Seq 
libraries were prepared using the TruSeq RNA Sample Preparation Kit (Illumina).

Libraries were sequenced on an Illumina HiSeq 2000 platform at the Australian

Genome Research Facility (AGRF) in Melbourne, Australia. Sequencing produced a total of 189.5 million individual 100 bp paired-end reads (Table 1 ).

\section{Processing of Illumina data}

The raw Illumina reads were filtered and adapters were clipped using TRIMMOMATIC (v0.32) (Bolger et al., 2014). Reads were filtered based on quality and size as follows; both universal and indexed Illumina adapters were clipped, quality trimming was also performed by removing leading and trailing bases with Phred quality score $<25$ and average Phred quality score was calculated in 4 bp sliding windows. Bases were trimmed from the point in the read where average Phred quality score dropped below 20 (i.e. the chances that a base is called incorrectly is 1 in 100) and reads of $<50$ bp were also excluded.

\section{De novo assembly and annotation of transcriptome}

The trimmed/filtered Illumina reads were used for de novo transcriptome assembly using Trinity (r20140717 version). The assembly was carried out with the recommended protocol described in (Haas et al., 2013) and using options appropriate for de novo transcriptome assembly of strand specific RNA-Seq libraries. Minimum contig length of 500 and read normalization were specified. Trinity collects transcripts with shared sequence identity into clusters that are loosely related to genes. The longest isoform per cluster was selected using a custom Perl script from the assembled Trinity output "assembled transcriptome" for the purpose of 
annotation. Chromera contigs were annotated by similarity search using batch

BLASTX conducted locally against the Swiss-Prot protein database downloaded in September 2014 (E-value cut off $10^{-3}$ and maximum 20 hits). Raw BLASTX outputs were imported to Blast2GO suite (version 2.6.5) (http://blast2go.com/b2ghome) for functional annotation and Gene Ontology (GO) assignment. KEGG analysis was also performed using the KEGG Automatic Annotation Server (KAAS) (Moriya et al., 2007) (http://www.genome.jp/kaas-bin/kaas main) in order to obtain an overview of the associated metabolic pathways. The bi-directional best hit $(\mathrm{BBH})$ method was used to obtain KEGG orthology (KO) assignments.

In order to validate the accuracy of the de novo assembly, reads were mapped back to the de novo-assembled transcriptome using the BOWTIE aligner version 0.12.7 (Langmead and Salzberg, 2012) with default mapping parameters. The percent of the mapped reads as proper pairs was used to assess the assembly quality. Moreover, BLASTN (E-value of $\leq 10^{-10}$ ) was performed against bacterial genomes downloaded from the GenBank, NCBI to determine the percentages of putative bacterial transcripts in the dataset. The completeness of the transcriptome assembly was assessed using BUSCO v3.1.0 making use of the function run_BUSCO.py (-mode transcriptome) with the Eukaryota_odb9, alveolate_stramenophile and protists_ensembl data (retrieved 23 October 2019).

\section{Phylogenomic analyses}

A multi-gene phylogenetic analysis was performed to assess the relative phylogenetic distance between Chromera strains and infer their evolutionary 
relationship to other Apicomplexans and Symbiodiniaceae. The protein sequences of GBR Chromera were predicted by Transdecoder (Haas et al., 2013). Transcript nucleotide (CDS) and protein sequences for Chromera CCMP2878 strain "Sydney Chromera" were downloaded from CryptoDB (release-37; http://cryptodb.org/cryptodb/), and for Plasmodium falciparum from PlasmoDB (release-37; http://plasmodb.org/plasmo/). Corresponding data for Symbiodiniaceae species were based on gene models from their respective genome sequencing projects; specifically, Fugacium kawagutii (Lin et al 2015) was obtained from the Symka Genome Database (http://web.malab.cn/symka_new/download.jsp), Cladocopium goreaui (Liu et al 2018) from ReFuGe 2020 site (http://refuge2020.reefgenomics.org/) and Breviolum minutum (Shoguchi et al., 2013) from the OIST Marine Genomics online resource (https://marinegenomics.oist.jp/symb/viewer/info?project id=21).

The longest transcript for each gene was extracted for all six species and used to infer orthologous clusters with OrthoFinder (Emms and Kelly, 2015). A total of 692 orthogroups were found to have representative genes in all species, and of these 172 consist of single-copy genes in each species; they represent strictly orthologous gene sets. Amino acid sequences for these 172 orthologous sets were aligned using MAFFT (Katoh and Standley, 2013) and converted into corresponding codon alignment by pal2nal (Suyama et al., 2006). Poorly aligned regions were removed using trimAl based on distribution of gaps (Capella-Gutierrez et al., 2009). Finally, IQTREE (Nguyen et al., 2015) was used to perform a partitioned phylogenetic analysis allowing independent estimation of evolutionary model for each protein set. To 
summarise the effect of phylogenetic relatedness on overlap between gene repertoires the number of shared orthologs was calculated for all members of each clade based on orthogroup information generated with OrthoFinder (see above). Additionally, for each pair of taxa the orthogroup information was used to compute a Hammings distance based on the ratio of the number of unshared orthogroups (those unique to either taxon in the pair) to the total number of orthogroups shared by the pair.

\section{Comparative transcriptomics}

Transcriptomes of Sydney Chromera, Fugacium, and the known mutualistic Symbiodiniaceae (Cladocopium and Breviolum) were also mapped against the KEGG database as previously performed for the GBR strain (using the bi-directional best hit (BBH) method). Genes mapped to different KEGG categories were calculated and compared. To better understand relationships between corals and both Chromera and Fugacium, repertoires of genes in categories that are important for symbiosis, such as $A B C$ transporters, as well as those involved in processes such as nitrogen metabolism and stress tolerance, were compared. The overlap amongst these genes was plotted using the R package UpSetR https://github.com/hmsdbmi/UpSetR/ (Conway et al., 2017).

\section{Results and discussion}

GBR Chromera transcriptome assembly and annotation 
After confirming the identity of Chromera cultures using novel Chromera-specific PCR primers, a transcriptome assembly was generated from 166 million paired-end Illumina reads ( 33 million per library; Table 2$)$. The number of putative genes (39 457, based on the longest transcript isoform per Trinity gene cluster) identified in the GBR Chromera isolate (Table 2) is comparable to those predicted for various Symbiodiniaceae isolates (30 000-49 000) based on transcriptome and genome data (Bayer et al., 2012;Shoguchi et al., 2013;Rosic et al., 2015;Aranda et al., 2016; Liu et al. 2018 and Shoguchi et al., 2018), but is higher than the number predicted from the Chromera genome of the reference strain (26 112 excluding TEs; Woo et al., 2015). As only $19.4 \%$ of the GBR Chromera genes had significant BLASTX hits against the Swiss-Prot protein database, the majority of Chromera genes code for unknown functions. This level of novelty is to be expected for organisms such as chromerids that are evolutionarily distant from well-characterized species, and has previously also been observed with Symbiodiniaceae and other dinoflagellates (Lin et al., 2010;Bayer et al., 2012; Stephens et al., 2018). To assess the quality of the de novo assembled transcriptome, reads were mapped to the assembly and an average of $84 \%$ of the paired Illumina reads were mapped successfully (Table 1 ). The assembled transcriptome was judged to be relatively comprehensive on the basis of high percentages of reads mapping.

5225 of Swiss-Prot annotated genes (68.35\%) were assigned to $38271 \mathrm{GO}$ terms. Biological process (GO-BP) accounted for the majority of GO terms (22 205, $58.02 \%$ ), followed by cellular component (GO-CC; 11 149, 29.1\%) and molecular function (GO-MF; 4 917, 12.8\%). Functions involved in cellular process and 
metabolic process (16\% and $14 \%$, respectively) were highly represented amongst GO-BP. In GO-MF, the most represented terms were catalytic activity (46\%) followed by binding (36\%). In GO-CC, the terms cell (39\%) and organelle (33\%) were highly represented (Supporting information Figure 2). Most of the KEGG-based annotations (34\% of all assignments) were assigned to the metabolic pathway category, followed by the human disease category (18\% of all assignments) (Supporting information Figure 3). Moreover, signal transduction and infectious diseases were the most highly represented pathways (Supporting information Figure 4, Table 2).

Several different approaches were used in order to assess the completeness of the assembled transcriptome. The KEGG annotation was searched for essential protein complexes/ pathways and the majority of genes for the pathways were found. Searched complexes included core cellular/ molecular protein complexes and pathways such as Ribosome biogenesis in eukaryotes, Ribosome, RNA polymerase, Spliceosome and Proteasome (Supporting information Table 3 and Figures 5-9). The use of BUSCO analysis resulted in a moderately high recovery $(\sim 60 \%)$ of conserved eukaryotic genes and relatively low recovery ( 26-46\%) of conserved alveolate and protist genes, respectively, using default settings (Supporting information Table 4). In addition, $0.1 \%$ of the assembled Chromera sequences (contigs) had BLASTN hits to bacterial databases $\left(\mathrm{E}\right.$-value $\leq 10^{-10}$ ) indicating very low bacterial contamination.

Sequence and functional similarities of the two Chromera strains

Phylogenomic analyses based on 172 orthologous single copy genes revealed a close relationship (branch length 0.07 ) between the GBR and Sydney strains of Chromera compared with divergences between different species within the 
Symbiodiniaceae (branch lengths 1.23 to 2.27) (Fig.1). Pairwise nucleotide similarity

between the Sydney and GBR Chromera isolates was $96.2 \%$, whereas the corresponding figures for the genera of Symbiodiniaceae were 72-78\%. A close relationship between the Chromera isolates was further supported by the presence of a relatively large number (14645) of shared orthologous genes and an average nucleotide identity of $99.12 \%$ based on alignments between these one-to-one orthologs. The overall distributions of the six main KEGG categories were similar in the two Chromera strains, one third of KEGG-annotated genes being assigned to metabolism (Supporting information Figure 10). This might reflect broadly similar functions and lifestyles. The only attempt to understand the nature of the coralChromera association used the Sydney strain (Mohamed et al., 2018). Given the high level of similarity between the GBR and Sydney harbour strains, the responses of corals to them are unlikely to differ significantly.

Focus on genes implicated in the symbiotic lifestyle To gain additional perspectives on the coral-Chromera interaction, the assembled transcriptomes for the Chromera strains were compared to those for Fugacium (which is assumed to be non-mutualistic) and to those of Cladocopium and Breviolum, which are mutualistic Symbiodiniacae strains, focusing on categories of genes likely involved in symbiosis, particularly nitrogen metabolism, transport and stress.

Metabolic exchanges in coral-algal symbioses 
Nutrient exchange between the symbiotic partners is of major importance in coralSymbiodinium mutualisms (Meyer and Weis 2012, Davy et al., 2012, Lin et al., 2015, Aranda et al., 2016). However, the nature of the translocated material(s) and mechanisms underlying exchanges between the coral host and algal symbionts are still unclear. To assess the potential for nutrient exchange between Chromera and coral hosts, the representation of the KEGG pathways Nitrogen metabolism and ATP-binding cassette $(A B C)$ transporters were investigated in the two Chromera strains and compared to those in the three members of the Symbiodiniaceae (Fugacium, Cladocopium and Breviolum).

The Nitrogen metabolism category was investigated on the basis that nitrogen cycling or conservation appears to be critical to the coral-Symbiodinium mutualism. Nitrogen is thought to be a growth limiting factor in nutrient-poor tropical waters, and many marine microbes have the ability to assimilate inorganic nitrogen (Pernice et al., 2012), for a recent review see Radecker at al. (2015). The results of a survey of genes captured under the KEGG pathway identifier KO00910 (Nitrogen metabolism) are presented in Supporting information Table 4. Note that this KEGG pathway does not include ammonium transporters, although it has previously been reported that the genomes of Symbiodiniaceae encode multiple ammonium transport proteins (Aranda et al., 2016).

In ocean waters, nitrate is generally the most abundant form of available nitrogen, concentrations often being at least an order of magnitude higher than those of ammonium and nitrite. However, in hospite, ammonium is likely to be the dominant 
nitrogen source available to intracellular symbionts. Thus, facultative symbionts (as most Symbiodiniaceae are thought to be) must not only be nutritionally versatile, but also able to regulate genes involved in the transport and assimilation of different $\mathrm{N}$ sources. As might be expected, all of the algae surveyed encode nitrate /nitrite transporters as well as enzymes required for assimilation of nitrogen in these forms. Although nitrate/nitrite transporters (NRTs) were present in all of the algae surveyed, some differences were apparent. Whereas the NRTs of Breviolum, Fugacium and Chromera were the MFS-type (K02575), this type was not detected in Cladocopium; rather, in this organism, components (K15577 and K15579) of a distinct ABC-type NRT were found. This difference may be significant; in cyanobacteria, the MFS-type has high affinity for both nitrate and nitrite, whereas the ABC-type has a much higher affinity for nitrate (Maeda and Omata, 2009).

\section{ATP-binding cassette $(A B C)$ transporters}

The $A B C$ class includes the largest number of transporters involved in either or both uptake and export of a wide range of substrates, including inorganic ions, carbohydrates and lipids. $\mathrm{ABC}$ transporters have been implicated in translocation of nutrients and metabolites in cnidarian symbioses (Davy et al., 2012; Mathews et al., 2017; Mohamed et al., 2019), and hence were an obvious focus for comparative analyses.

As with the nitrogen metabolism category, surveying $A B C$ transporter complements (Fig. 2) was largely unsuccessful in providing general molecular criteria by which known mutualists (Breviolum and Cladocopium) can be distinguished from 
commensals or parasites. One potentially significant difference, however, is that both Breviolum and Cladocopium encode K10111 members, which are nominal transporters of a variety of different sugars and which may be involved in carbohydrate translocation in hospite, whereas proteins of this type were not detected in either Fugacium or Chromera (Supporting information Table 5). Unfortunately, the survey provides few other grounds for speculation about photosynthate translocation. Whilst a number of candidates for roles in sterol or lipid translocation were detected, these were generally not restricted to the coral mutualists. For example, components of the mla/lin type transport system for phospholipids/sterols/gamma-HCH (K02065 and K02066) were detected in Fugacium as well as Cladocopium, and the Chromera ABC repertoire does include possible sterol transporters (K05683, K05681, K08712).

Despite these analyses being uninformative on the issue of general characteristics of mutualists, they do provide grounds for speculation about the near-ubiquitous association of Cladocopium with Indo-Pacific corals (LaJeunesse et al., 2018) and particularly with Acropora spp.. The ABC repertoire of Cladocopium by far exceeded those of all of the other organisms surveyed (Fig. 2), and included many genes identified only in this organism, amongst which were transporters for sugars, lipids and amino acids as well as inorganic nutrients. A range of candidate amino acid transporters were restricted to Cladocopium, one of which (HisP) is of particular interest in the context of coral symbioses, as it encodes a histidine transport ATPbinding protein (Supporting information Table 6). The Cladocopium strain included here was originally isolated from Acropora tenuis (Liu et al., 2018), a member of the 
complex coral superfamily, and whereas robust corals (members of the other coral superfamily) have a complete histidine biosynthetic pathway, complex corals (like all other animals) must acquire this either from the diet or from their resident endosymbionts (Ying et al., 2018). Also intriguing in the context of mutualism is the detection only in Cladocopium of three components required for cystine transport (K02424, K10009, K10010); the interest in these stems from the fact that, amongst corals, members of the genus Acropora lack one of the enzymes required for cysteine biosynthesis (Shinzato et al., 2011). In this respect, Acropora appears to be unique; all other corals surveyed had a compete cysteine pathway. The presence of an efficient cystine transport system may therefore underlie the near ubiquitous association of Cladocopium $\mathrm{C} 1$ with Acropora spp.

\section{Fugacium - an evolutionary recidivist?}

The ABC transporters identified in Fugacium include several candidates for roles in nutrient exchange - for example, K05641 encodes an ABCA1 protein known as the cholesterol efflux regulatory protein (CERP) that mediates efflux of cellular cholesterol and phospholipids (reviewed by Zhao and Lappalainen, 2012). Note that an ABCA1 gene was up-regulated in adult Acropora millepora colonies in the light, i.e. when transport of photosynthate from alga to host occurs (Bertucci et al.,2015). K02065, which was present in all three Symbiodiniaceae, encodes a phospholipid/cholesterol/gamma-HCH transport system ATP-binding protein, again with a potential function in lipid/sterol translocation. Indeed, some ABC family A transporters (ABCA7 and $\mathrm{ABCA}$ ) were upregulated in Cladocopium during establishment of symbiosis with coral larvae (Mohamed et al., 2019). Thus, although 
Fugacium is now considered not to be a coral mutualist, the comparative analyses presented here do not rule this out. Whilst the nature of the relationship between Fugacium and corals is still unclear, the apparent contradictions could, however, be rationalized by consideration of the phylogenetic position of Fugacium. The ability to become endosymbiotic is considered to be a defining characteristic of the family Symbiodiniaceae (LaJeunesse et al., 2018); as a member of a derived clade within the Symbiodiniaceae, Fugacium may have undergone (or be undergoing) secondary loss of symbiotic potential but its genome still bear vestiges of this ancestral condition.

The small heat shock protein HSP20 might explain stress tolerance in Chromera Molecular chaperones are important for refolding damaged proteins (Vierling, 1991), thus their involvement in stress tolerance is inevitable. Amongst these chaperones we looked at heat shock proteins in both Chromera and two symbiotic members of Symbiodiniaceae, Cladocopium and Breviolum. Despite genes coding for chaperones in the KEGG Orthology 03110 Chaperones and folding catalysts (including HSP90/70) being present in similar numbers in all of these genera, gene(s) coding for two HSP20 isoforms were found in both Chromera strains to the exclusion of the three Symbiodiniaceae algae (Fig. 3). Consistent with a potential role in stress tolerance the HSP20 was reported recently to be correlated with stress tolerance in reef-building corals where coral species containing more HSP20 genes were more stress tolerant (robust corals) than those with smaller numbers of HSP20 genes (complex corals) (Ying et al., 2018). 


\section{Conclusions}

This paper describes de novo assembly and annotation of a transcriptome for a Chromera strain isolated from a GBR coral, which was generated in order to understand diversity within the species and to provide a better understanding of the metabolic capabilities and lifestyle of this photosynthetic apicomplexan alga. The GBR strain has a high degree of similarity with the Sydney (culture collection) strain, hence infection studies based on the former (e.g. Mohamed et al., 2018) can be generalized to the latter. Comparisons between Chromera, the Symbiodiniaceae algae Fugacium, Cladocopium and Breviolum and the apicomplexan parasite $P$. falciparum based on specific categories of genes were inconclusive with respect to common molecular characteristics of mutualists, but suggest that HSP20 genes may underlie the higher thermal tolerance of Chromera compared to Symbiodiniaceae. The presence of specific genes implicated in mutualism suggest that Fugacium may have secondarily lost the ability to establish symbioses, and the comparative analyses provide a molecular rationale for the near-ubiquitous association of Cladocopium with Indo-Pacific corals.

\section{Acknowledgments}

The research was supported by the Australian Research Council through Grant CE140100020 to DM via the ARC Centre of Excellence for Coral Reef Studies at James Cook University. AM was supported by PhD scholarships provided by the Cultural Affairs and Mission sectors of the Egyptian Ministry of Higher Education and Scientific Research and James Cook University Postgraduate Research Scholarship 
(JCUPRS) and through the AIMS@JCU joint- scheme. The authors gratefully thank

Dr Vivian Cumbo at Macquarie University for supplying Chromera cultures.

\section{Conflict of Interest}

The authors declare that they have no conflict of interest.

DATA AVAILABILITY. Data reported in this study have been submitted to the NCBI Gene Expression Omnibus (GEO) under accession number GSE139820 including raw illumina data, transcriptome assembly and annotations.

\section{Author contributions}

AM and DM conceived and designed the study. AM conducted the experiments and generated the RNA-Seq data. AM performed bioinformatics required for transcriptome assembly, annotation and comparative analyses with guidance from CXC and MR. JZ and IC performed the phylogenomic analysis. AM, EB and DM interpreted that data and wrote the manuscript. All authors read the article and approved the final version.

\section{Table legends}

Table 1. Illumina sequencing and mapping statistics: number of reads and bases of raw and processed data after quality control for each library and percentages of reads successfully mapped to the GBR Chromera transcriptome. 
Table 2. Summary statistics of the GBR Chromera transcriptome assembly using Trinity and annotation carried out based on the Swiss-Prot (SP), Gene Ontology (GO) and KEGG databases

\section{Figure legends}

Figure 1 (A) Unrooted phylogenetic tree based on maximum-likelihood phylogenetic analysis of 172 orthologous nucleotide sequences from Sydney Chromera, GBR Chromera, $P$. falciparum, F. kawagutii, B. minutum and C. goreaui. All nodes in the tree were fully supported (100\% of bootstraps) based on 1000 bootstraps by ultrafast bootstrap in IQtree. The sizes of filled circles at some nodes in the tree indicate numbers of shared othologs. (B) Heat map summarizing Hamming distances between taxa based on nucleotide similarity in the 172 orthologous sequences used for phylogeny reconstruction.

Figure 2 UpsetR plot (which allows comparative visualisation of the presence/absence of genes of a pathway in different taxa) illustrating the intersection between genes of two KEGG pathways: (A) Nitrogen metabolism (KO00910) and (B) ABC transporters (KO02010) in the two Chromera strains, Fugacium, mutualistic Symbiodinianceae (Cladocopium and Breviolum) and the parasite $P$. falciparum. Each plot is generated from a binary matrix with unique KEGG identifiers for each pathway and each taxon in a column. The number of genes per taxon is represented by the length of the orange bars. The black dots represent presence/absence. The purple columns show the number of genes shared amongst 
taxa or exclusive to one of them. So, reading the upper plot from the left there are 8 different genes (each gene is represented once) that are exclusively found in Cladocopium and not the others, second column shows 3 genes that are found in the 3 symbiodiniaceae and the 2 Chromera, but not present in $P$. falciparum, the third column shows 3 genes that are found in all taxa considered, etc. The intersection size reflects the number of unique or shared genes.

Figure 3 Comparison of the repertoires of genes encoding chaperones and folding catalysts (KO03110) of Chromera strains and Symbiodiniaceae. (A) A five-way Venn diagram of the gene overlap amongst the two Chromera strains, mutualistic Symbiodiniaceae (Cladocopium and Breviolum) and Fugacium. (B) A bar graph illustrating numbers of HSP20, HSP70 and HSP90 genes identified in the five algal datasets.

\section{References}

Aranda, M., Li, Y., Liew, Y.J., Baumgarten, S., Simakov, O., Wilson, M.C. et al. (2016). Genomes of coral dinoflagellate symbionts highlight evolutionary adaptations conducive to a symbiotic lifestyle. Sci Rep 6: 39734.

Barott, K.L., Rodriguez-Brito, B., Janouškovec, J., Marhaver, K., Smith, J.E., Keeling, P., et al. (2011) Microbial diversity associated with four functional groups of benthic reef algae and the reef-building coral Montastraea annularis. Environ Microbiol 13:1192-204.

Bayer, T., Aranda, M., Sunagawa, S., Yum, L.K., DeSalvo, M.K., Lindquist, E. et al. (2012). Symbiodinium Transcriptomes: Genome Insights into the Dinoflagellate Symbionts of ReefBuilding Corals. Plos One 7.

Bertucci, A., Foret, S., Ball, E.E., and Miller, D.J. (2015) Transcriptomic differences between day and night in Acropora millepora provide new insights into metabolite exchange and light - enhanced calcification in corals. Mol Ecol, 24: 4489-4504. 
Bolger, A.M., Lohse, M., and Usadel, B. (2014). Trimmomatic: a flexible trimmer for Illumina sequence data. Bioinformatics 30: 2114-2120.

Capella-Gutierrez, S., Silla-Martinez, J. M., and Gabaldon, T. (2009). trimAl: a tool for automated alignment trimming in large-scale phylogenetic analyses. Bioinformatics 25 : 1972-1973. doi: 10.1093/bioinformatics/btp348

Chakravarti, L.J., Negri, A.P. and van Oppen, M. (2019) Thermal and Herbicide Tolerances of Chromerid Algae and Their Ability to Form a Symbiosis with Corals. Front Microbiol 10: 173. https://doi.org/10.3389/fmicb.2019.00173

Chen, H., and Boutros, P.C. (2011) VennDiagram: a package for the generation of highly customizable Venn and Euler diagrams in R. BMC Bioinformatics 12: 35.

Clerissi, C., Brunet, S., Vidal-Dupiol, J., Adjeroud, M., Lepage, P., Guillou, L., et al. (2018). Protists within corals: the hidden diversity. Front Microbiol 9: 2043. doi:

10.3389/fmicb.2018.02043

Conway, J.R., Lex, A., and Gehlenborg, N. (2017) Upsetr: An r package for the visualization of intersecting sets and their properties. Bioinformatics 33: 2938-40.

Cumbo, V.R., Baird, A.H., Moore, R.B., Negri, A.P., Neilan, B.A., Salih, A. et al. (2013). Chromera velia is Endosymbiotic in Larvae of the Reef Corals Acropora digitifera and A. tenuis. Protist 164: 237-244.

Davy, S.K., Allemand, D., and Weis, V.M. (2012) Cell biology of cnidarian - dinoflagellate symbiosis. Microbiol and Mol Biol Rev 76: 229-261.

Emms, D. M., and Kelly, S. (2015). OrthoFinder: solving fundamental biases in whole genome comparisons dramatically improves orthogroup inference accuracy. Genome Biol. 16: 157.

Gonzalez-Pech, R.A., Bhattacharya, D., Ragan, M.A., and Chan, C.X. (2019) Genome evolution of coral reef symbionts as intracellular residents. Trends Ecol Evol https://doi.org/10.1016/j.tree.2019.04.010

Haas, B.J., Papanicolaou, A., Yassour, M., Grabherr, M., Blood, P.D., Bowden, et al. (2013). De novo transcript sequence reconstruction from RNA-seq using the Trinity platform for reference generation and analysis. Nature Protocols 8: 1494-1512.

Howells, E.J., Beltran, V.H., Larsen, N.W., Bay, L.K., Willis, B.L., and Van Oppen, M.J. (2012) Coral thermal tolerance shaped by local adaptation of photosymbionts. Nature Climate Change 2: 116-120

Hughes, T. P., Kerry, J. T., Álvarez-Noriega, M., Álvarez-Romero, J. G., Anderson, K. D., Baird, A. H. et al. (2017). Global warming and recurrent mass bleaching of corals. Nature 543: 373-377. doi: 10.1038/nature21707

Janouškovec, J., Horak, A., Barott, K.L., Rohwer, F.L., and Keeling, P.J. (2012) Global analysis of plastid diversity reveals apicomplexan-related lineages in coral reefs. Curr Biol. 22: R518-R519. 
Janouškovec, J., Horák, A., Barott, K. L., Rohwer, F. L., and Keeling, P. J. (2013).

Environmental distribution of coral-associated relatives of apicomplexan parasites. ISME J.

7: 444-447. doi: 10.1038/ismej.2012.129

Katoh, K., and Standley, D. M. (2013). MAFFT multiple sequence alignment software version 7: improvements in performance and usability. Mol. Biol. Evol 30: 772-780. doi:

$10.1093 / \mathrm{molbev} / \mathrm{mst010}$

Kwong, W. K., Campo, J., del Mathur, V., Vermeij, M. J. A., and Keeling, P. J. (2019). A widespread coral-infecting apicomplexan contains a plastid encoding chlorophyll biosynthesis. Nature. 568:103-107. doi: 10.1038/s41586-019-1072-z.

LaJeunesse, T. C., Parkinson, J. E., Gabrielson, P. W., Jeong, H. J., Reimer, J. D., Voolstra, C. R., and Santos, S.R. (2018). Systematic revision of Symbiodiniaceae highlights the antiquity and diversity of coral endosymbionts. Curr Biol, 28: 2570-2580.

Langmead, B., and Salzberg, S. L. (2012). Fast gapped-read alignment with Bowtie 2. Nature methods 9: 357.

Lin, S., Zhang, H., Zhuang, Y., Tran, B., and Gill, J. (2010). Spliced leader-based metatranscriptomic analyses lead to recognition of hidden genomic features in dinoflagellates. Proc Nat Acad Sci USA 107: 3-20038.

Lin, S., Cheng, S., Song, B., Zhong, X., Lin, X., Li, W. et al. (2015). The Symbiodinium kawagutii genome illuminates dinoflagellate gene expression and coral symbiosis. Science 350: 691-694.

Liu, H., Stephens, T.G., González-Pech, R., Beltran, V.H., Lapeyre, B., Bongaerts, P. et al. (2018) Symbiodinium genomes reveal adaptive evolution of functions related to coraldinoflagellate symbiosis. Comm Biol 1: 95.

Maeda, S-I., and Omata, T. (2009) Nitrite transport activity of the ABC-type cyanate transporter of the cyanobacterium Synechococcus elongatus. J Bacteriol 191: 3265-3272.

Mathur, V., del Campo, J., Kolisko, M., Keeling, P.J. (2018). Global diversity and distribution of close relatives of apicomplexan parasites. Environ Microbiol doi:10.1111/1462-

$2920.14134 \mathrm{~ms}$

Matthews, J.L., Crowder, C.M., Oakley, C.A., Lutz, A., Roessner, U., Meyer, E., Grossman, A.R. et al. (2017) Optimal nutrient exchange and immune responses operate in partner specificity in the cnidarian-dinoflagellate symbiosis. Proc Nat Acad Sci USA 114:1319413199.

Meyer, E., and Weis, V.M. (2012) Study of cnidarian - algal symbiosis in the "omics" age. Biol Bull 223:44-65.

Mohamed, A.R., Cumbo, V., Harii, S., Shinzato, C., Chan, C.X., Ragan, M.A. et al. (2016) The transcriptomic response of the coral Acropora digitifera to a competent Symbiodinium strain: the symbiosome as an arrested early phagosome. Mol Ecol 25:3127-41. 
Mohamed, A.R., Cumbo, V.R., Harii, S., Shinzato, C., Chan, C.X., Ragan, M.A. (2018). Deciphering the nature of the coral-Chromera association. Isme $\mathrm{J} 12: 776-790$.

Mohamed, A.R., Andrade, N., Moya, A., Chan, C.X., Negri, A.P., Bourne, D.G. et al. (2019). Transcriptomic insights into the establishment of coral-algal symbioses from the symbiont perspective. BioRxiv, 652131. https://doi.org/10.1101/652131.

Moore, R.B., Obornik, M., Janouskovec, J., Chrudimsky, T., Vancova, M., Green, D.H. et al. (2008). A photosynthetic alveolate closely related to apicomplexan parasites. Nature 451: 959-963.

Moriya Y, Itoh M, Okuda S, Yoshizawa AC, Kanehisa M (2007). KAAS: an automatic genome annotation and pathway reconstruction server. Nucleic acids research 35: W182-185.

Nguyen, L.T., Schmidt, H. A., Von Haeseler, A., and Minh, B.Q. (2015). IQ-TREE: a fast and effective stochastic algorithm for estimating maximum-likelihood phylogenies. Mol Biol Evol 32:268-274. doi: $10.1093 / \mathrm{molbev} / \mathrm{msu} 300$

Oborník M, Vancová M, Lai DH, Janous $\square$ kovec J, Keeling PJ, Lukes $\square$ J. Morphology and ultrastructure of multiple life cycle stages of the photosynthetic relative of apicomplexa. Chromera velia Protist. 2011;162:115-30.

Pernice, M., Meibom, A., Van Den Heuvel, A., Kopp, C., Domart-Coulon, I., Hoegh-Guldberg, O., et al. (2012). A single-cell view of ammonium assimilation in coral-dinoflagellate symbiosis. ISME J. 6:1314-1324. doi: 10.1038/ismej.2011.196

Rädecker, N., Pogoreutz, C., Voolstra, C.R., Wiedenmann, J., and Wild, C. (2015). Nitrogen cycling in corals: the key to understanding holobiont functioning? Trends Microbiol 23:490497. doi: $10.1016 /$ j.tim.2015.03.008

Rosic, N., Ling, E.Y., Chan, C.K., Lee, H.C., Kaniewska, P., Edwards, D. et al. (2015) Unfolding the secrets of coral-algal symbiosis. ISME J 9:844-856.

Shinzato, C., Shoguchi, E., Kawashima, T., Hamada, M., Hisata, K., Tanaka, M., et al. (2011). Using the Acropora digitifera genome to understand coral responses to environmental change. Nature 476:320-323.

Shoguchi, E., Shinzato, C., Kawashima, T., Gyoja, F., Mungpakdee, S., Koyanagi, R. et al. (2013). Draft assembly of the Symbiodinium minutum nuclear genome reveals dinoflagellate gene structure. Curr Biol 23:1399-1408.

Shoguchi, E., Beedessee, G., Tada, I., Histata, K., Kawashima, T., Takeuchi T. et al (2018) Two divergent Symbiodinium genomes reveal conservation of a gene cluster for sunscreen biosynthesis and recently lost genes. BMC Genomics 19:458.

Stephens, T.G., Ragan, M.A., Bhattacharya, D., Chan, C.X. (2018) Core genes in diverse dinoflagellate lineages include a wealth of conserved dark genes with unknown functions. Sci Reports 8: 17175. 
Suyama, M., Torrents, D., and Bork, P. (2006). PAL2NAL: robust conversion of protein sequence alignments into the corresponding codon alignments. Nucleic Acids Res. 34: W609-W612.

Vierling, E. (1991) The roles of heat shock proteins in plants. Ann Rev Plant Biol 42:579-620.

Visser, P., Bintoudi, E., Boschker, E., Frade, P., van Bleijswijk, J., and Matthijs, H. (2012).

Newly discovered coral endosymbiont Chromera is more thermotolerant than Symbiodinium. in 12th International Coral Reef Symposium Abstracts (Cairns), 198. Available online at: www.icrs2012.com

Voolstra, C.R., Schwarz, J.A, Schnetzer, J., Sunagawa, S., Desalvo, M.K., Szmant, A.M, et al. (2009) The host transcriptome remains unaltered during the establishment of coral-algal symbioses. Mol Ecol. 18:1823-33.

Woo, Y.H., Ansari, H., Otto, T.D, Klinger, C.M., Kolisko, M., Michalek, J. et al. (2015). Chromerid genomes reveal the evolutionary path from photosynthetic algae to obligate intracellular parasites. eLife 4: e06974.

Ying, H., Cooke, I., Sprungala, S., Wang, W., Hayward, D.C., Tang, Y., et al. (2018) Comparative genomics reveals the distinct evolutionary trajectories of the robust and complex coral lineage. Genome Biol. 19:175

Zhao, H., and Lappalainen, P. (2012). A simple guide to biochemical approaches for analyzing protein-lipid interactions. Mol Biol Cell 23, 2823-2830. doi: 10.1091/mbc.E11-070645 

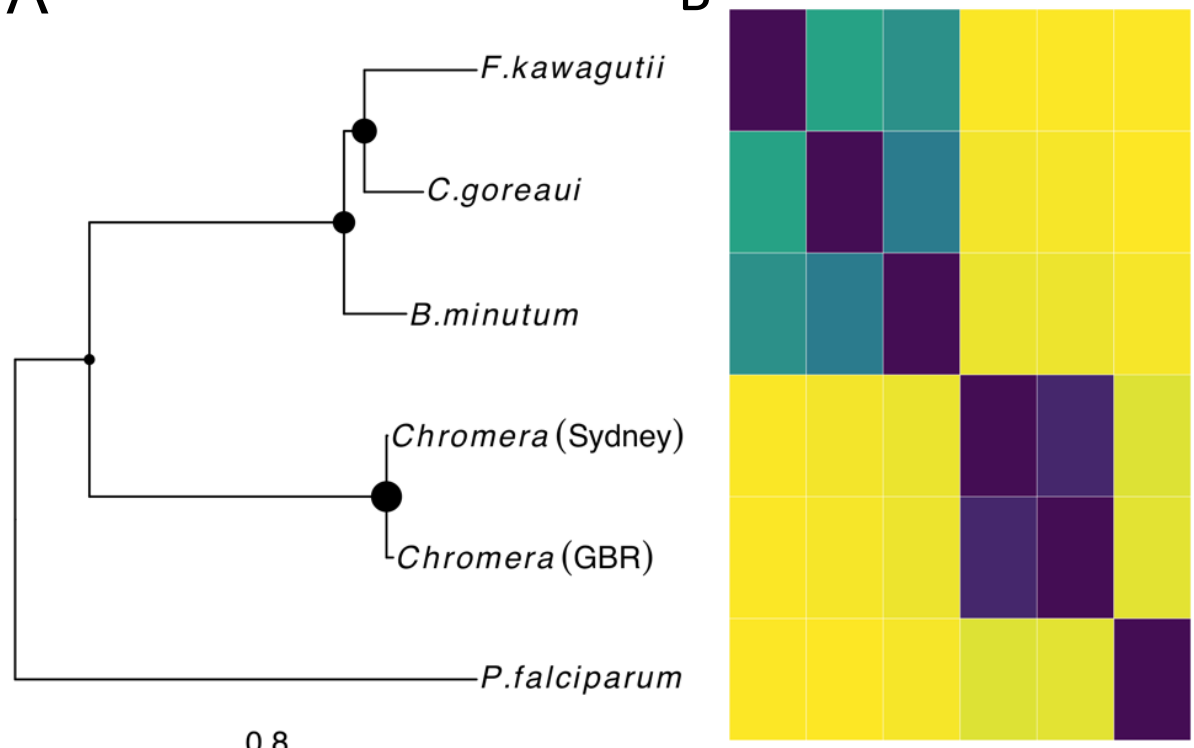

Shared Orthologs

- 5000

10000

15000

Hamming Distance

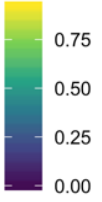

0.8

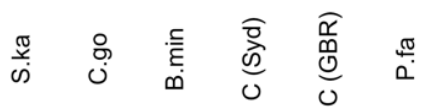




\section{A) Chaperones and folding catalysts}

\section{8 molecular chaperones in both Chromera}

1. HSP20 family protein

2. stress 70 protein chaperone microsome-associated protein

3. Dnas homolog subfamily B member 8

4. molecular chaperone $\mathrm{HscB}$

5. STIP1 homology and U-box containing protein 1

6. major intracellular serine protease (isp)

7. serine protease

8. minor extracellular serine protease $\mathrm{Vpr}$

\section{B) Heat shock protein genes}

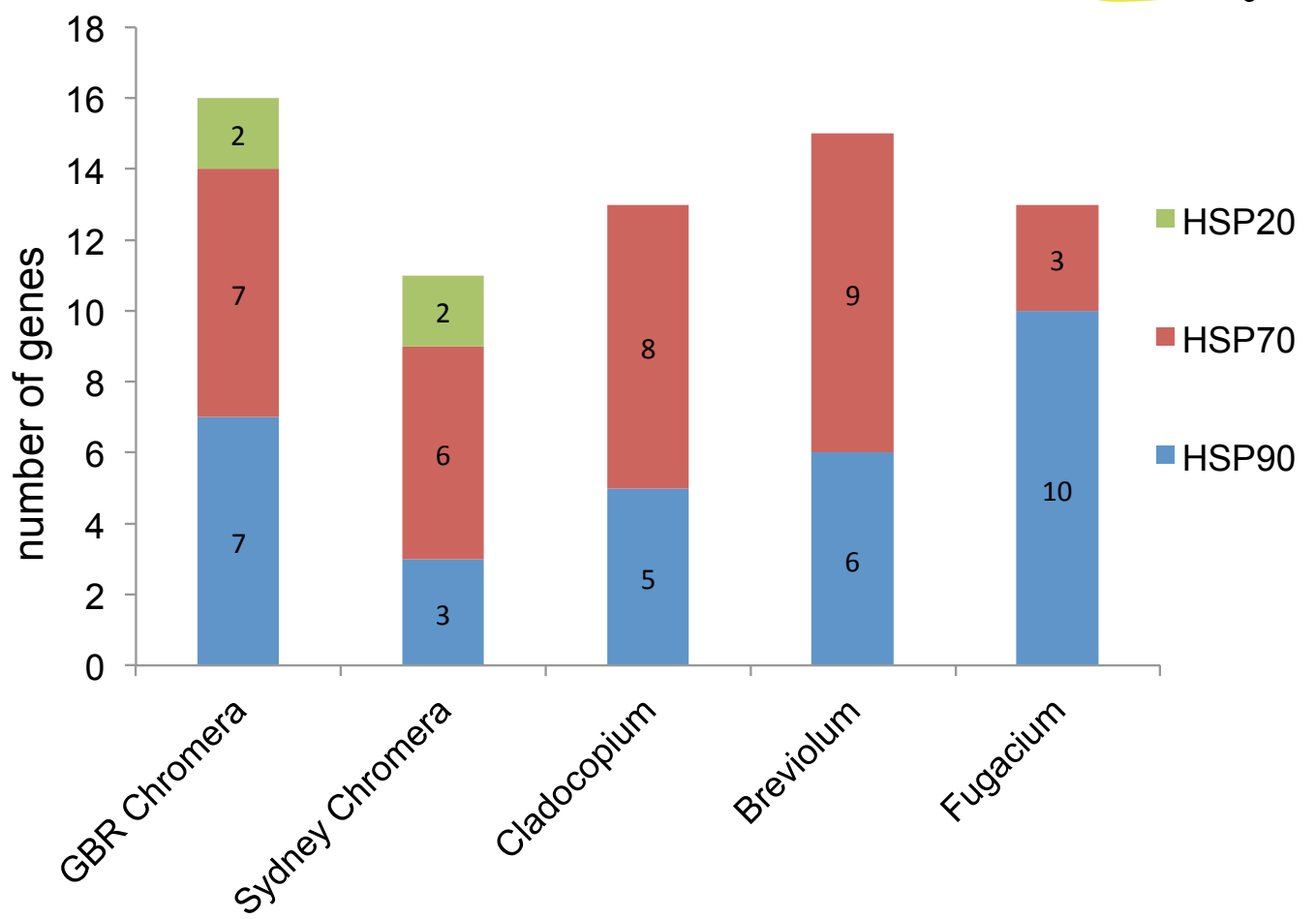

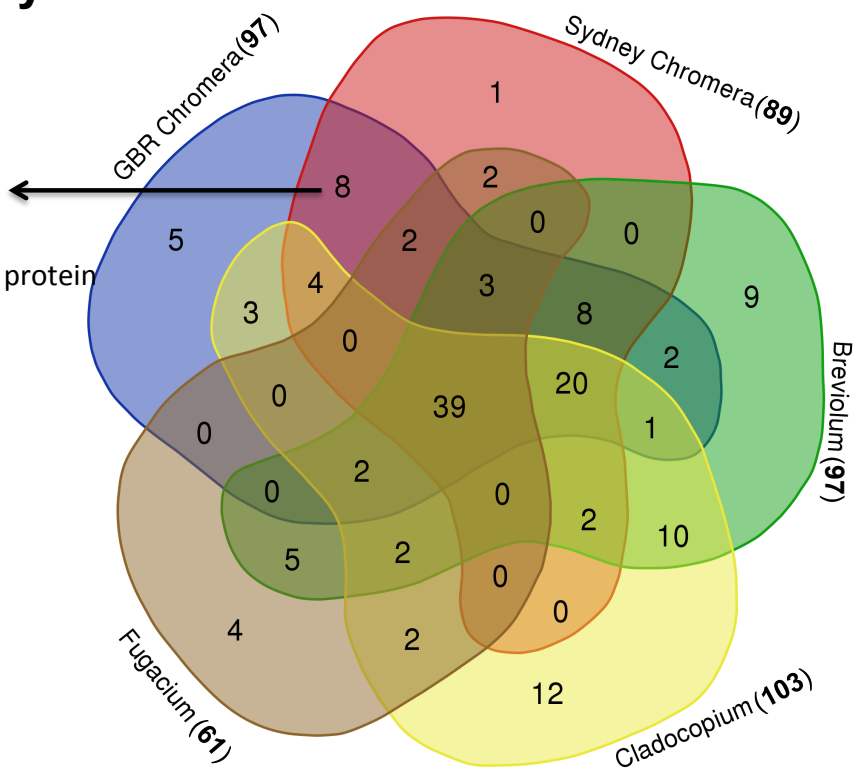




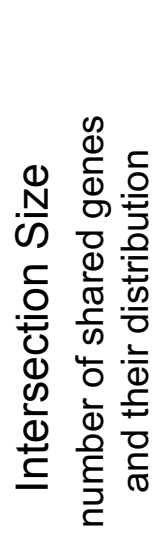

A) Nitrogen metabolism

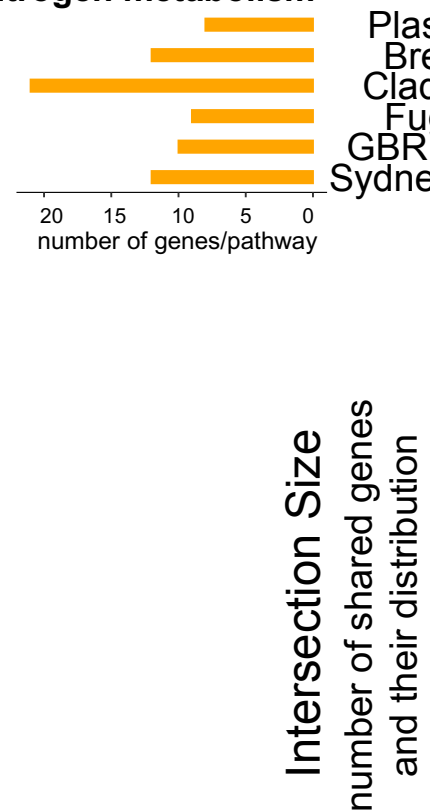

B) ABC transporters

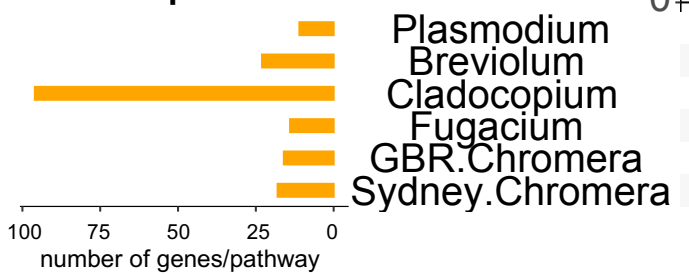
lasmodium Breviolum Cladocopium Fugacium GBR.Chromera dney.Chromera $\begin{array}{llll}20 & 15 & 10 & 5\end{array}$
20.
0.0
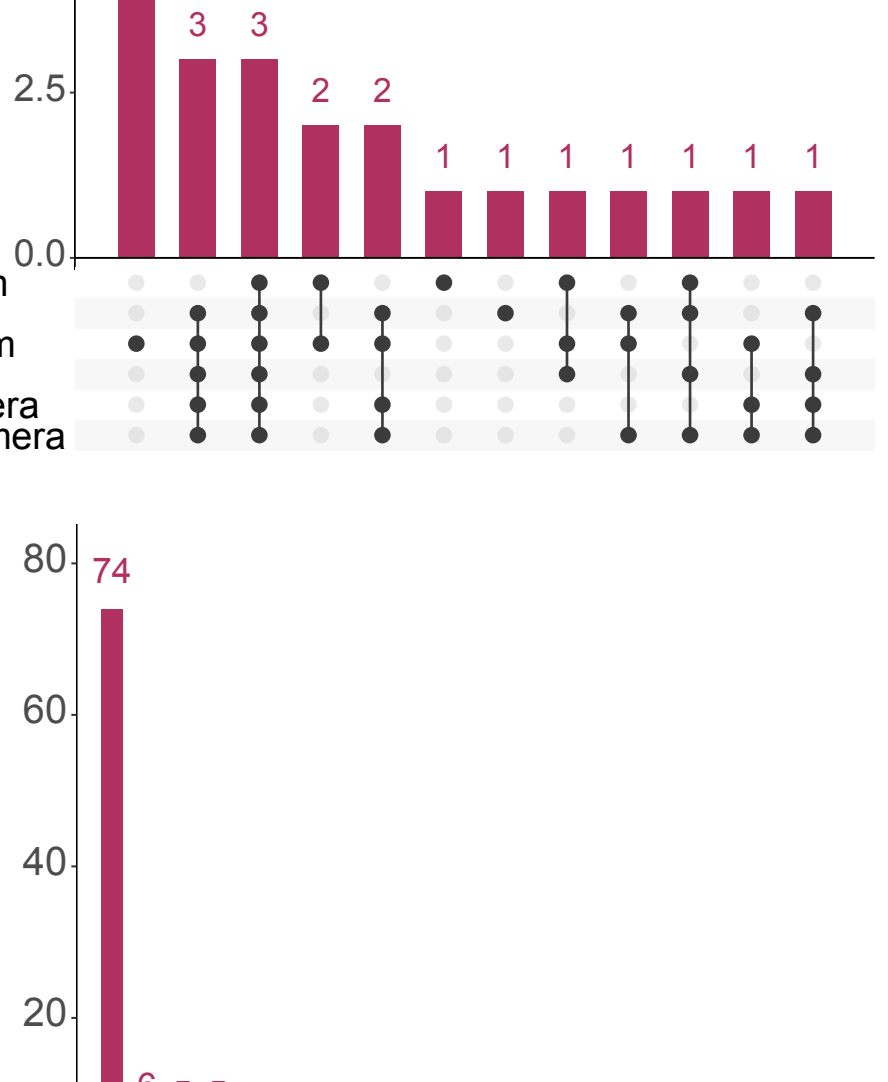

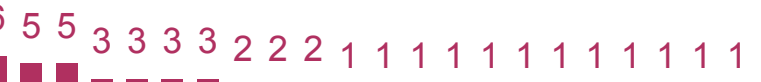

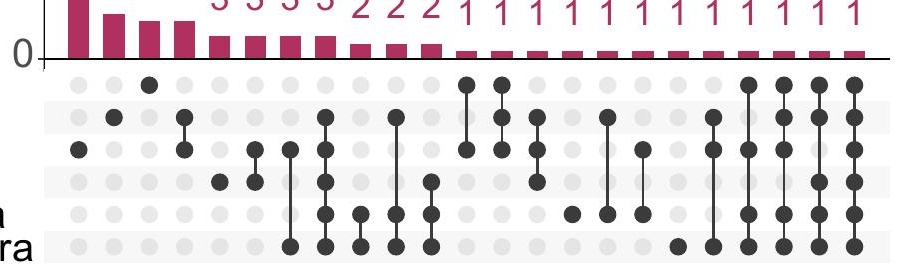


Table 1. Illumina sequencing and mapping statistics: number of reads and bases of raw and processed data after quality control (adapter removal, trimming and filtering low-quality bases) for each library and percentages of reads successfully mapped onto the GBR Chromera transcriptome. Note that Chromera was subjected to a variety of treatments prior to RNA extraction in order to ensure that the transcriptome assembly captured as many genes as possible.

\begin{tabular}{|c|c|c|c|c|c|c|c|c|}
\hline \multicolumn{9}{|c|}{ RNA-Seq library/ culturing condition } \\
\hline & Control & Cold & Heat & Dark & Motile & Mixotrophic & Total & Average \\
\hline No. raw paired-end reads $(\mathrm{M})$ & 31.35 & 31.75 & 31.49 & 31.8 & 30.17 & 32.91 & 189.47 & 31.57 \\
\hline No. raw bases $(\mathrm{Gb})$ & 6.27 & 6.35 & 6.29 & 6.36 & 6.03 & 6.58 & 37.88 & 6.31 \\
\hline No. reads passed $\mathrm{QC}(\mathrm{M})$ & 27.59 & 28.03 & 27.46 & 27.9 & 26.47 & 28.93 & 166.38 & 27.73 \\
\hline No. bases passed QC (Gb) & 5.51 & 5.6 & 5.49 & 5.58 & 5.29 & 5.78 & 33.25 & 5.54 \\
\hline Mapping rate (\%) & 85.2 & 83.9 & 86.9 & 83.7 & 81.3 & 82.5 & - & 84.2 \\
\hline
\end{tabular}


Table 2. Summary statistics for the GBR Chromera de novo transcriptome assembly using Trinity and annotation based on Swiss-Prot (SP), Gene Ontology (GO) and KEGG databases

\begin{tabular}{|l|l|}
\hline Trinity outputs: & 39457 \\
\hline Total Trinity 'genes' & 79842 \\
\hline Total Trinity transcripts & 53.42 \\
\hline GC \% & 2289 \\
\hline Statistics based on ALL transcript contigs: \\
\hline Contig N50 & 1461 \\
\hline Median contig length & 1838.03 \\
\hline Average contig & 146752195 \\
\hline Total assembled bases & 2220 \\
\hline Statistics based on ONLY LONGEST ISOFORM per 'GENE': \\
\hline Contig N50 & 1403 \\
\hline Median contig length & 1764.26 \\
\hline Average contig & 69612282 \\
\hline Total assembled bases & 7644 \\
\hline Annotation statistics based on longest isoform per gene data: \\
\hline Swiss-Prot (SP) database & 5225 \\
\hline Gene Ontology (GO) database & 4220 \\
\hline KEGG database &
\end{tabular}

